\title{
ACBE, a new base editor for simultaneous C-to-T and A-to-G substitutions in mammalian systems
}

Jingke Xie ${ }^{1,2,3 \dagger}$, Xingyun Huang ${ }^{1,3 \dagger}$, Xia Wang ${ }^{1,4}$, Shixue Gou ${ }^{1,3}$, Yanhui Liang ${ }^{1,3}$, Fangbing Chen 1,3, Nan Li ${ }^{1,2,3}$, Zhen Ouyang ${ }^{1,2,4}$, Quanjun Zhang ${ }^{1,2,4}$, Weikai Ge ${ }^{1,2,3}$, Qin Jin ${ }^{1,2,3}$, Hui Shi ${ }^{1,3}$, Zhenpeng Zhuang ${ }^{1,3}$, Xiaozhu Zhao ${ }^{1,3}$, Meng Lian ${ }^{1,5}$, Jiaowei Wang ${ }^{1,3}$, Yinghua Ye ${ }^{1,2,4}$, Longquan Quan ${ }^{1,2,4}$, Han $\mathrm{Wu}^{1,2,4}$, Kepin Wang ${ }^{1,2,4^{*}}$ and Liangxue Lai ${ }^{1,2,4^{*}}$

\begin{abstract}
Background: Many favorable traits of crops and livestock and human genetic diseases arise from multiple single nucleotide polymorphisms or multiple point mutations with heterogeneous base substitutions at the same locus. Current cytosine or adenine base editors can only accomplish C-to-T (G-to-A) or A-to-G (T-to-C) substitutions in the windows of target genomic sites of organisms; therefore, there is a need to develop base editors that can simultaneously achieve C-to-T and A-to-G substitutions at the targeting site.

Results: In this study, a novel fusion adenine and cytosine base editor (ACBE) was generated by fusing a heterodimer of TadA (ecTadA ${ }^{W T / *}$ ) and an activation-induced cytidine deaminase (AID) to the $\mathrm{N}$ - and C-terminals of Cas9 nickase (nCas9), respectively. ACBE could simultaneously induce C-to-T and A-to-G base editing at the same target site, which were verified in HEK293-EGFP reporter cell line and 45 endogenous gene loci of HEK293 cells. Moreover, the ACBE could accomplish simultaneous point mutations of C-to-T and A-to-G in primary somatic cells (mouse embryonic fibroblasts and porcine fetal fibroblasts) in an applicable efficiency. Furthermore, the spacer length of sgRNA and the length of linker could influence the dual base editing activity, which provided a direction to optimize the ACBE system.
\end{abstract}

Conclusion: The newly developed ACBE would expand base editor toolkits and should promote the generation of animals and the gene therapy of genetic diseases with heterogeneous point mutations.

Keywords: Adenine and cytosine base editor (ACBE), Simultaneous C-to-T and A-to-G conversions, Mammalian systems

\footnotetext{
*Correspondence: wang_kepin@gibh.ac.cn; lai_liangxue@gibh.ac.cn

${ }^{+}$Jingke Xie and Xingyun Huang contributed equally to this work.

'CAS Key Laboratory of Regenerative Biology, Guangdong Provincial Key Laboratory of Stem Cell and Regenerative Medicine, Guangzhou Institutes of Biomedicine and Health, Chinese Academy of Sciences, Guangzhou 510530, China

Full list of author information is available at the end of the article
}

(c) The Author(s). 2020 Open Access This article is licensed under a Creative Commons Attribution 4.0 International License, which permits use, sharing, adaptation, distribution and reproduction in any medium or format, as long as you give appropriate credit to the original author(s) and the source, provide a link to the Creative Commons licence, and indicate if changes were made. The images or other third party material in this article are included in the article's Creative Commons licence, unless indicated otherwise in a credit line to the material. If material is not included in the article's Creative Commons licence and your intended use is not permitted by statutory regulation or exceeds the permitted use, you will need to obtain permission directly from the copyright holder. To view a copy of this licence, visit http://creativecommons.org/licenses/by/4.0/ The Creative Commons Public Domain Dedication waiver (http://creativecommons.org/publicdomain/zero/1.0/) applies to the data made available in this article, unless otherwise stated in a credit line to the data. 


\section{Background}

The rapid development of gene editing technologies (ZFNs, TALENs, and CRISPR/Cas9) plays an increasingly important role in biomedical and agricultural fields [1]. Many desirable agricultural traits of crops and livestock and human genetic diseases arise from multiple single nucleotide polymorphisms (SNPs) or multiple point mutations with heterogeneous base substitutions at the same locus [2-7]. Therefore, base editing of a genome in multiple sites with heterogeneous base substitutions is necessary to achieve favorable traits in agriculture, establish human disease animal models, and treat human hereditary diseases $[8,9]$. Genome editing with either the CRISPRbased cytosine base editor (CBE) or the adenine base editor $(\mathrm{ABE})$ can be used for C-to-T or A-to-G base substitutions in a high efficiency, but both editors are not applicable for correction of other variants such as base transconversion, small insertions, and deletions (indels) [10-12]. Compared with base editors, the prime editing system (PE), a "search-and replace" genome editing tool, can induce base substitutions in more extended regions [13]. However, the efficiency of PE for making transition point mutations was reported much lower than that of both $\mathrm{CBE}$ and $\mathrm{ABE}$, which makes PE difficult to be used to generate organisms or correct genetic diseases that need base editing of a genome in multiple sites with heterogeneous base substitutions [13-15].

In an earlier report, Li et al. fused APOBEC3A-ecTadAecTadA7.10 or ecTadA-ecTadA7.10-APOBEC3A, to the $\mathrm{N}$ terminus of nCas9 (D10A), together with UGI at the C terminus of nCas9 (D10A), and successfully induced C-to$\mathrm{T}$ and A-to-G conversions simultaneously at the same target site in plants [16]. A preprint (recently published in Nature Biotechnology) presents a novel base editor, Target-ACE, which integrates the abilities of ABEs and CBEs and can simultaneously induce C-to- $\mathrm{T}$ and A-to-G base editing in a mammalian immortalized cell line, human embryonic kidney HEK293Ta cells [17]. However, Target-ACE was not verified in primary somatic cells and other mammalian cells, which is vital for applying the base editor for generation of animals through the somatic cell nuclear transfer approach and correction of human genetic diseases. A version of CBEs [11], namely, Target-AID, has two unique features: one is that the main editing window of protospacer positions is within 1-5 instead of 4-8 as that in other APOBEC1 based CBEs and the second is that PmCDA1, the cytidine deaminase used in this system, is fused to the $\mathrm{C}$ terminus of Cas9 nickase (nCas9) instead of the $\mathrm{N}$ terminus. By contrast, $\mathrm{A} \cdot \mathrm{T}$ base pair to $\mathrm{G} \cdot \mathrm{C}$ base pair conversions in an $\mathrm{ABE}$ system are performed within the editing window of protospacer positions $4-8$, and adenine deaminase, a heterodimer of $\operatorname{TadA}\left(\right.$ ecTadA $\left.^{\mathrm{WT} /{ }^{\prime \prime}}\right)$, is fused to the $\mathrm{N}$ terminus of nCas9 [12]. The differences of the position of deaminases and the editing windows of deaminases between target-AID and ABE make them complementary with each other in terms of structure. Therefore, in this study, we fused Target-AID and ABE7.10 to generate a new base editing tool, namely, $A C B E$. We verified that $A C B E$ could simultaneously generate C-to-T (G-to-A) and A-to-G (T-to-C) not only in the target sites of an immortalized cell line (HEK293 cells), but also in primary somatic cells such as mouse embryonic fibroblasts (MEFs) and porcine fetal fibroblasts (PFFs). Moreover, we confirmed that the efficiency of simultaneous C-to-T and A-to-G conversions could be improved through optimization of linker length and sgRNA spacer length. Dual-function ACBE would expand the toolkit of base editors and has the potential biomedical and agricultural applications.

\section{Results}

Evaluation of the function of ACBE in a HEK293-EGFP cell line

The PmCDA1 and UGI of Target-AID were fused to the $C$ terminus of $A B E-7.10$, an optimized version of the $\mathrm{ABE}$ system, with a nuclear localization signal (NLS) located at the $\mathrm{N}$ and $\mathrm{C}$ terminals to engineer a new base editor with the abilities of Target-AID and ABE-7.10 (Fig. 1a). To verify whether the size of base editor expression cassettes $(\mathrm{CBE}, \mathrm{ABE}$, and $\mathrm{ACBE}$ ) influence the expression levels of base editors, additionally, base editor expressing vectors fused to the base editor with enhanced green fluorescent protein (EGFP) by P2A were constructed (Additional file 1: Fig. S1a). The HEK293 cells were transfected with Plasmids CBE-EGFP $(7.2 \mu \mathrm{g})$, ABE-EGFP $(7.2 \mu \mathrm{g})$, and ACBE-EGFP $(8 \mu \mathrm{g})$, respectively. Two-day post transfection, the cells were collected and analyzed by flow cytometry. The results showed that the proportion of EGFP-positive cells (52.1\%, 52.0\%, and $55.7 \%$ ) and the EGFP intensity had no significant differences among the three groups, indicating that the size of base editors did not influence the expression levels (Additional file 1: Fig. S1b-d).

Next, we demonstrated whether ACBE could simultaneously generate C-to- $\mathrm{T}$ and A-to-G conversions in the same target site. An HEK293-EGFP cell line expressing an EGFP, which is under the control of an endogenous $h$ Rosa 26 promoter, was used to test the dual base editing function of ACBE [18]. Two EGFP-targeting sgRNAs, namely, EGFP-Stop1 and EGFP-Stop2, which corresponded to Target-AID and ABE-7.10, respectively, were designed and constructed (Fig. 1b). Generation of a premature stop codon and disruption of the start codon in the EGFP gene would be performed by C-to- $\mathrm{T}$ conversion (EGFP-Stop1) and A-to-G substitution (EGFPStop2), respectively, and both point mutations were expected to block the EGFP expression of HEK293-EGFP cells. The ACBE vector and their corresponding sgRNAs 


\begin{tabular}{|c|c|c|c|c|c|c|c|c|c|c|c|c|}
\hline $\begin{array}{l}\text { CBE } \\
\text { (Target -AID) }\end{array}$ & pCMV & nCas9 (D10A) & NLS & $10 a a$ & $\mathrm{SH} 3$ & CDA1 & & 9aa & UGI & NLS & & \\
\hline ABE7.10 & pCMV & ecTadAWT/* & 32aa & & Cas9 (D10A) & & NLS & & & & & \\
\hline ACBE & CMV & ecTadAWT/* & 32aa & & Cas9 (D10A) & & NLS & 10aa & $\mathrm{SH} 3$ & CDA1 & 9aa & UGI \\
\hline
\end{tabular}

C
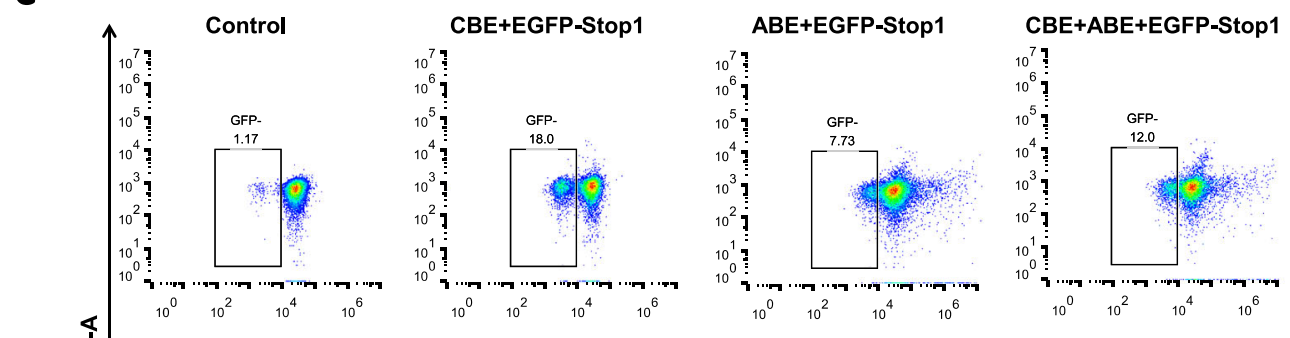

ACBE+EGFP-Stop1

CBE+EGFP-Stop2

ABE+EGFP-Stop2

CBE+ABE+EGFP-Stop2
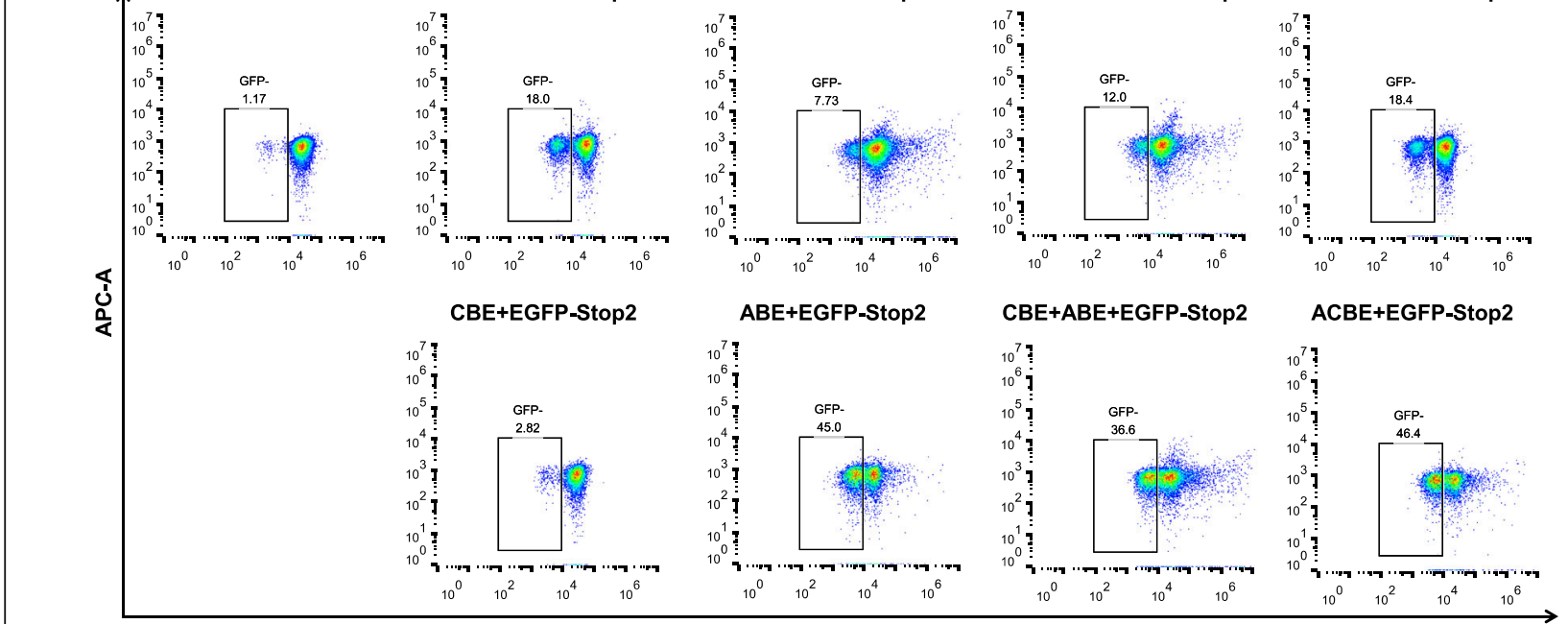

b

CGG

EGFP

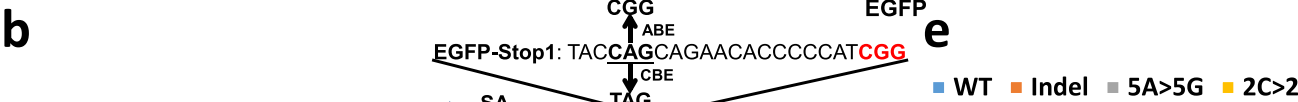

$\|$ WT $=$ Indel $\| 5 A>5 G=2 C>2 T=5 A>5 G / 2 C>2 T=O$ Other
CBE+EGFP-Stop2
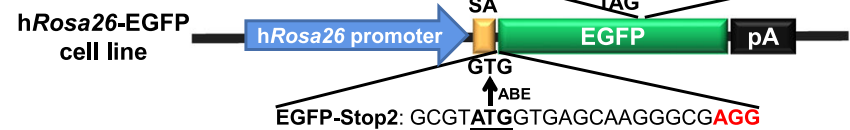

d

d EGFP-Stop1

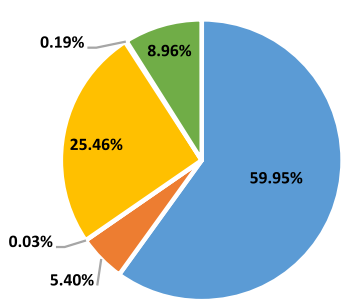

$0.22 \% \quad 0.26 \% \Gamma^{3.40 \%}$

EGFP-Stop2
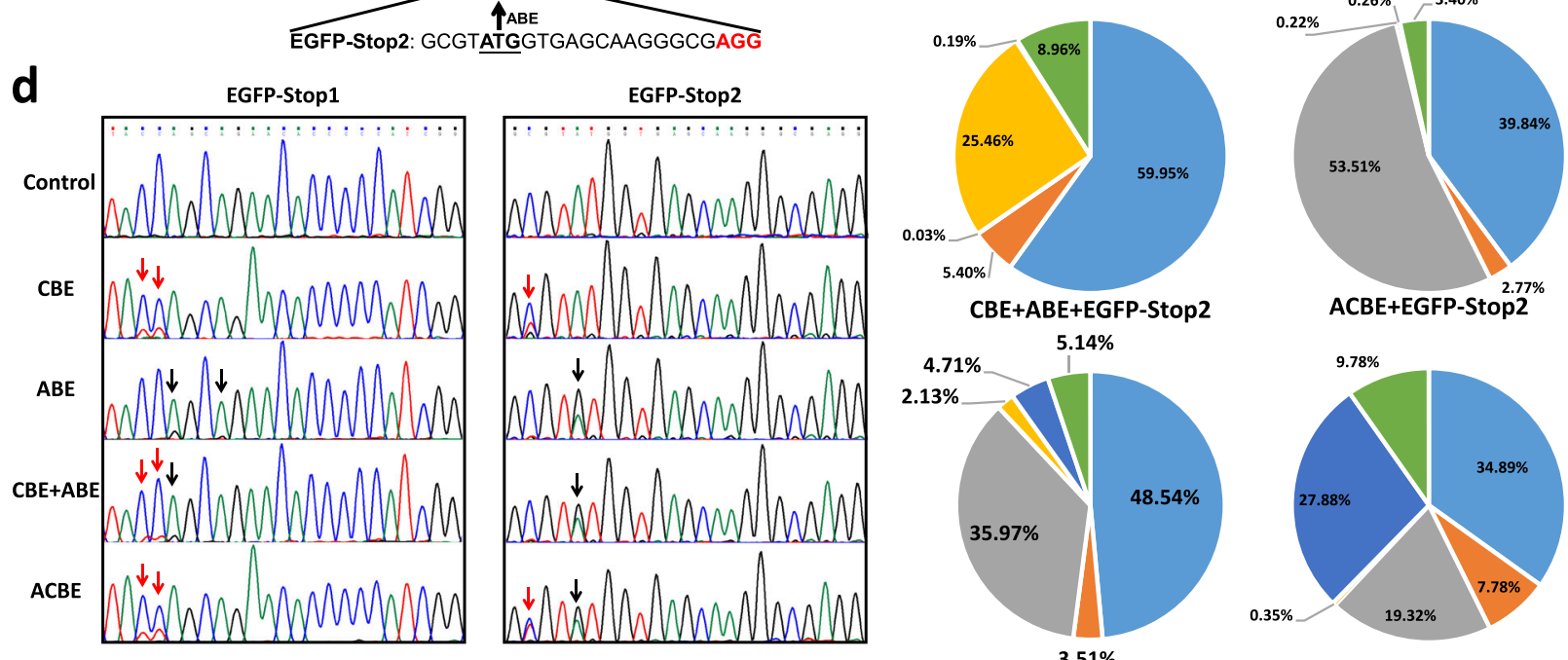

ACBE+EGFP-Stop2

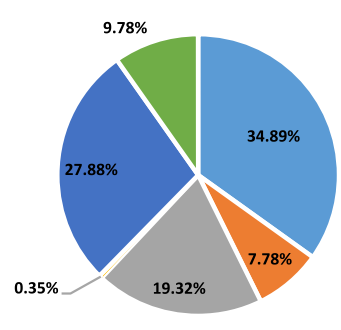

Fig. 1 Simultaneous C-to-T and A-to-G base substitutions induced by the ACBE system. a Architectures of the dual-function base editing system, ACBE, and individual base editing systems, namely Target-AID and ABE7.10. ecTadA ${ }^{\mathrm{WT} / *}$, a heterodimer of an evolved Escherichia coli TadA and a wild-type TadA; aa, amino acid; NLS, nuclear localization signal; UGI, inhibitor of uracil DNA glycosylase. b Schematic of the HEK293-EGFP reporter cell line and two targeting sgRNA (EGFP-Stop1 and EGFP-Stop2), which could induce a stop codon and mutation of start codon, respectively. c The representative results of flow cytometry showed the proportion of EGFP-negative cells edited by different base editors with the corresponding sgRNA. Three independent experiments were performed. $\mathbf{d}$ The representative Sanger sequencing results of the cell samples transfected with different base editors showed the target base substitutions in the targeting sites EGFP-Stop1 and EGFP-Stop2. The C-to-T and Ato-G substitutions were marked with red and black arrows, respectively. e The editing effects on the target site EGFP-Stop2 generated by the $\mathrm{ACBE}$ and $\mathrm{CBE}+\mathrm{ABE}$ system. Different parts with various colors in the pie chart showed the frequencies of the corresponding mutation type 
were co-electroporated into HEK293-EGFP cells. The transfection of Target-AID (referred to as CBE in figures) or ABE7.10 (referred to as $\mathrm{ABE}$ in figures) was used as a negative control. In addition, the previous report showed that $\mathrm{ABE}$ combined with $\mathrm{CBE}$ could introduce A-to-G and C-to- $\mathrm{T}$ substitutions simultaneously in mouse embryos [19]. Target-AID plus ABE7.10 (referred to as $\mathrm{CBE}+\mathrm{ABE}$ in figures) were used as a positive control. The cells were collected 5 days after transfection and flow cytometry assay was performed to detect the proportion of the EGFP-negative cells. As shown in Fig. 1c and Additional file 1: Fig. S2a, the EGFP expression was silent in $18.4 \%$ of the cells when the ACBE was transfected with EGFP-Stop1, and this proportion was almost equal to that resulted from $\mathrm{CBE}(18.0 \%)$ and $\mathrm{CBE}+\mathrm{ABE}$ (12.0\%). Moreover, 7.73\% EGFP-positive cells were observed in bulks of HEK293-EGFP cells transfected with ABE and EGFP-Stop1 probably because EGFP-Stop1 and ABE-mediated missense point mutation (Q184R) shifted the wavelength of excitation light or the intensity of green fluorescence [20]. When ACBE was transfected with EGFP-Stop2, the EGFP expression was silent in $42.5 \%$ of the cells, similar to that resulted from $\mathrm{ABE}(45.0 \%)$ and $\mathrm{CBE}+\mathrm{ABE}$ (36.6\%). These results indicated that the ACBE system independently maintained CBE and ABE functions in human cells. The collected cell samples were further subjected to Sanger sequencing. The results showed that simultaneous C-to$\mathrm{T}$ substitution at position 2 and A-to-G substitution at position 5 were observed in the ACBE-transfected HEK293-EGFP cells at the EGFP-Stop2 target site, but not in the CBE + ABE transfected HEK293-EGFP cells. Although simultaneous $\mathrm{C}$-to- $\mathrm{T}$ substitutions at positions 3 and 4, and A-to-G substitution at position 5 occurred in the CBE + ABE transfected HEK293-EGFP cells at the EGFP-Stop1 target site, only C-to- $\mathrm{T}$ substitutions at positions 3 and 4 were found in the ACBE-transfected cells (Fig. 1d and Additional file 1: Fig. S2b, 2c). Whether these simultaneous substitutions of these two loci occurred in the same DNA strand, polymerase chain reaction (PCR) products were analyzed by TA-cloning sequencing. The Sanger sequencing results of TAcloning confirmed that only the cell samples cotransfected EGFP-Stop2 with ACBE showed simultaneous C-to- $\mathrm{T}$ and A-to-G substitutions at the same DNA allele (Additional file 1: Fig. S2d). Amplicon deep sequencing results further confirmed that the efficiency of simultaneous C-to- $\mathrm{T}$ and A-to-G substitutions at the same DNA allele was $27.88 \%$ for ACBE at the EGFPStop2 target site, but significantly higher than that with the $\mathrm{CBE}+\mathrm{ABE}(4.71 \%)$ (Fig. 1e). Furthermore, the simultaneous C-to-T and A-to-G substitutions at the same DNA strand were almost not observed in cells transfected with either individual $\mathrm{CBE}$ (0.19\%) or $\mathrm{ABE}$
(0.22\%) (Additional file 1: Fig. S2e). These results indicated that the ACBE system could induce simultaneous C-to-T and A-to-G substitutions at the same target locus and create the heterologous mutation at the same DNA strand with high efficiency with single sgRNA.

\section{ACBE-mediated simultaneous C-to-T and A-to-G conversions in the endogenous loci of HEK293 cells}

Forty-five sgRNAs targeting seven human endogenous genes (15 for TP53, 4 for LMNA, 14 for PGK1, 3 for TDP43, 3 for CTNNB1, 2 for AAVS1, and 4 for $L D H A$ ) were designed and constructed (Additional file 2: Table S1). Forty-three of the sgRNAs covered cytosine and adenine at different positions of editing windows relative to the PAM sites, whereas two of them only had adenines, but not cytosines in their editing window of sgRNA. ACBE and sgRNA expressing vectors were cotransfected into wild-type HEK293 cells. Three-day post transduction, genomic DNAs were extracted and screened to determine whether simultaneous C-to- $\mathrm{T}$ and A-to-G conversions occurred at the target sites. PCR products surrounding the target sites were subjected to Sanger sequencing. Base editing frequency was quantified with EditR [21]. The results showed that 25 sgRNAs in the ACBE system could simultaneously achieve C-to$\mathrm{T}$ and A-to-G conversions (Fig. 2a). For the remaining 20 sgRNAs, 18 sgRNAs achieved only significant C-to-T base editing and 2 sgRNAs achieved only A-to-G base substitutions. Of these sgRNAs, 11 sgRNAs had no A in positions 4-6 and 2 sgRNAs had no $\mathrm{C}$ in positions $1-7$ (Additional file 2: Table S2). The average frequencies of C-to-T and A-to-G for every targeting nucleotide in different positions relative to PAM (referred to PAM as + 21 to $+23 \mathrm{bp}$ ) indicated that the region with a high efficiency of C-to- $\mathrm{T}$ conversion spanned from +1 to $+7 \mathrm{bp}$, ranging from 10.92 to $29.00 \%$, whereas that of A-to-G point mutation was from +4 to $+6 \mathrm{bp}$, with efficiencies ranging from 7.07 to $11.68 \%$ (Fig. 2b). The analysis of targeting sequences of 45 sgRNAs and their corresponding efficiencies of base editing showed that $\mathrm{ACBE}$ performed preference C-to- $\mathrm{T}$ conversions in TC motif, whereas no obvious motif preference for A-to-G point mutations (Additional file 1: Fig. S3). These results indicated that the ACBE had a similar spectrum and the editing efficiency in C-to- $\mathrm{T}$ conversions to Target-AID [11], whereas the narrowed editing spectrum and the reduced editing efficiency in A-to-G conversions were observed compared with that of ABE7.10 [12].

Five sgRNAs (P53-G7, P53-G8, LMNA-G1, LDHA-G1, and PGK1-G4) targeting four of the human endogenous genes (TP53, LMNA, LDHA, and PGK1) were selected to validate the base editing effects of the ACBE system on endogenous genes in detail. The Sanger sequencing results showed that the mutant peak of C-to- $\mathrm{T}$ was 


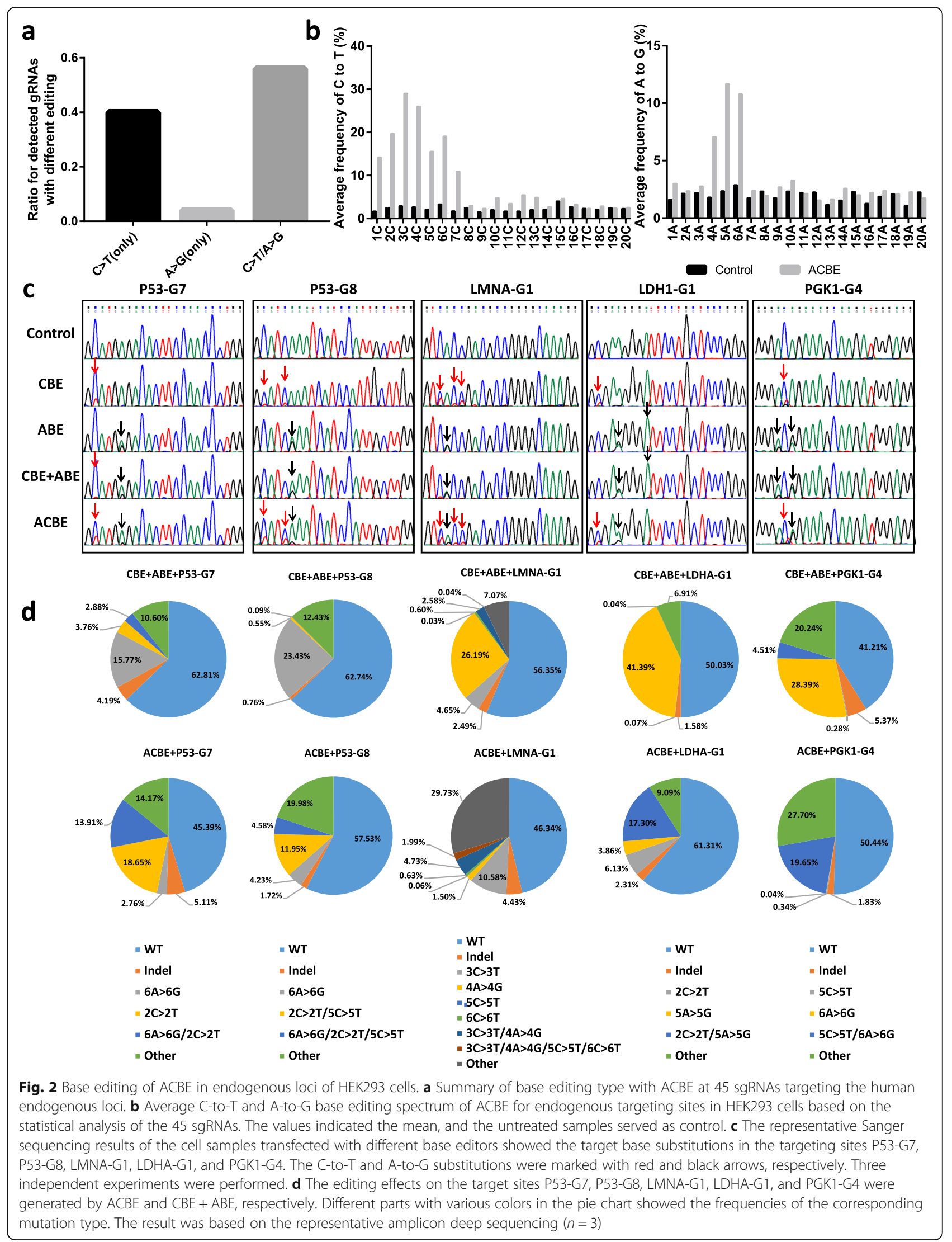


observed in the cells transfected with $\mathrm{CBE}$ and $\mathrm{ACBE}$ but not with $\mathrm{CBE}+\mathrm{ABE}$ (Fig. $2 \mathrm{c}$ and Additional file 1: Fig. S4). However, only A-to-G conversions were detected in cells transfected with $\mathrm{ABE}, \mathrm{CBE}+\mathrm{ABE}$, and $\mathrm{ACBE}$. These findings indicated that the ACBE system maintained an efficient dual functionality for $\mathrm{C}$-to- $\mathrm{T}$ and A-to-G mutation, consistent with the results of the reporter assay. Conversely, $\mathrm{CBE}+\mathrm{ABE}$ showed the prior editing of A-to-G and reduced $\mathrm{C}$-to- $\mathrm{T}$ editing. The $\mathrm{C}$ to- $\mathrm{T}$ base editing efficiency of ACBE was comparable with Target-AID, whereas the A-to-G editing efficiency was reduced compared with that of ABE7.10. Notably, the $\mathrm{CBE}+\mathrm{ABE}$ group showed a significantly reduced efficiency of C-to- $\mathrm{T}$ substitutions and maintained the normal function of A-to-G conversion among the five target sites, indicating that ABE7.10 might have better binding competitiveness than Target-AID to the same target DNA locus. Deep sequencing was conducted to analyze the effect of simultaneous C-to-T and A-to-G substitutions that occurred at a single target site of the same DNA strand by different base editing systems. The results revealed that the ACBE system could efficiently induce simultaneous heterogeneous base editing (A-to-G and C-to-T) at the same DNA strand with efficiencies of $13.91 \%(2 \mathrm{C}>2 \mathrm{~T}$ and $6 \mathrm{~A}>6 \mathrm{G})$ for P53-G7, 4.58\% $(2 \mathrm{C}>$ $2 \mathrm{~T}, 5 \mathrm{C}>5 \mathrm{~T}$, and $6 \mathrm{~A}>6 \mathrm{G})$ for $\mathrm{P} 53-\mathrm{G} 8,4.73 \%(3 \mathrm{C}>3 \mathrm{~T}$ and $4 \mathrm{~A}>4 \mathrm{G})$ and $1.99 \%(3 \mathrm{C}>3 \mathrm{~T}, 4 \mathrm{~A}>4 \mathrm{G}, 5 \mathrm{C}>5 \mathrm{~T}$, and $6 \mathrm{~A}>6 \mathrm{G})$ for LMNA-G1, $17.30 \%(2 \mathrm{C}>2 \mathrm{~T}$ and $5 \mathrm{~A}>$ 5G) for LDHA-G1, and $19.65 \%(5 \mathrm{C}>5 \mathrm{~T}$ and $6 \mathrm{~A}>6 \mathrm{G})$ for PGK1-G4, whereas the CBE + ABE system only had efficiencies of $2.88 \%$ for P53-G7, $0.09 \%$ for P53-G8, $2.58 \%$ and $0.04 \%$ for LMNA-G1, $0.04 \%$ for LDHA-G1, and $4.51 \%$ for PGK1-G4 (Fig. 2d). The individual base editor $\mathrm{CBE}$ or $\mathrm{ABE}$ almost could not induce simultaneous C-to-T and A-to-G substitution at the same DNA strand with a frequency below $0.23 \%$ in the five tested loci (Additional file 1: Fig. S5). The abovementioned results showed an advantage for ACBE to induce simultaneous C-to- $\mathrm{T}$ and A-to-G mutation at the same DNA strand. Except for heterogeneous base editing, homogeneous base editing (A-to-G or C-to-T) was also observed in the $\mathrm{CBE}+\mathrm{ABE}$ and $\mathrm{ACBE}$ groups (Fig. 2d, e). In addition, the proportions of indels formation in the five tested loci by different base editors were analyzed. The indels frequency of ACBE (1.72-5.11\%) was not increased compared with CBE (1.45-6.78\%), ABE (0.77$4.09 \%)$, and $\mathrm{CBE}+\mathrm{ABE}(1.58-5.37 \%)$, indicating that ACBE maintained the safe genome editing potential with a low frequency of DNA double-strand breaks (DSB) (Fig. 2d and Additional file 1: Fig. S5).

In addition, the off-target effects of ACBE on the potential off-target sites (POTs) were tested in the HEK293 gene loci. Eight off-target sites for P53-G7 and four offtarget sites for P53-G8 were selected to analyze the off- target effects between $\mathrm{ACBE}$ and individual base editor ( $\mathrm{CBE}$ and $\mathrm{ABE}$ ). From the Sanger sequencing results, no evident off-target effects were observed at POTs for $\mathrm{CBE}, \mathrm{ABE}$, and $\mathrm{ACBE}$ (Additional file 1: Fig. S6). Moreover, we tested the off-target effects of $\mathrm{ACBE}$ and $\mathrm{CBE}+$ ABE with LMNA-G1, LDHA-G1, and PGK1-G4. For each sgRNA, four top POTs were analyzed with Sanger sequencing. The results of the tested off-target sites showed no mutation peaks except for the site PGK1-G4OT1, which showed an evident A-to-G mutation peak induced by $\mathrm{CBE}+\mathrm{ABE}$ and a mild mutation peak induced by ACBE, indicating that ACBE had better specificity than $\mathrm{CBE}+\mathrm{ABE}$ with PGK1-G4 sgRNA (Additional file 1: Fig. S7). All the results of the abovementioned offtarget confirmed that the ACBE system showed high specificity in base editing, comparable to individual base editors.

\section{ACBE-mediated simultaneous C-to-T and A-to-G conversions in primary somatic cells}

In previous reports, dual-function base editors, saturated targeted endogenous mutagenesis editors (STEMEs), and Target-ACE, were verified the function of simultaneous C-to- $\mathrm{T}$ and A-to-G conversions in plants and HEK293Ta cell line, respectively [16, 17]. Whether simultaneous C-to-T and A-to-G substitutions could be induced in primary somatic cells and other mammalian cells was not validated. Next, the dual-function effects of ACBE were tested in primary somatic cells, including MEFs and PFFs. Four sgRNAs targeting the endogenous genes ( 3 for mstn and 1 for tyr) of MEFs and four sgRNAs targeting the endogenous genes ( 2 for $T Y R, 1$ for FANCA, and 1 for LMNA) of PFFs were designed and constructed (the sgRNA targeting sequences were shown in Additional file 2: Table S1). The individual sgRNA $(4 \mu \mathrm{g})$ with $\mathrm{CBE}+\mathrm{ABE}(7.2 \mu \mathrm{g}+7.2 \mu \mathrm{g})$ or ACBE $(8 \mu \mathrm{g})$ expressing vectors was co-transfected into the primary somatic cells. Three days after transfection, the cell samples were collected for amplicon deep sequencing. The read ratios of amplicon deep sequencing were used to represent the efficiencies of base editing (Fig. 3a, b). The results showed that ACBE-mediated simultaneous C-to- $\mathrm{T}$ and A-to-G point mutations occurred in three tested targeting loci of MEFs (M-mstnG1, G2, and M-tyr-G3) and four targeting loci of PFFs (P-TYR-G1, G3, P-FANCA-G1, and P-LMNA-AC1). Only a detectable A-to-G point mutation was observed for M-mstn-G4 because only suitable adenines were presented in the editing window of the ACBE system. However, for the $\mathrm{CBE}+\mathrm{ABE}$ system, simultaneous $\mathrm{C}$-to- $\mathrm{T}$ and A-to-G point mutations were only found at the PLMNA-AC1 targeting site, whereas only A-to-G substitutions occurred at other targeting loci. In addition, these results showed that the ACBE system maintained 


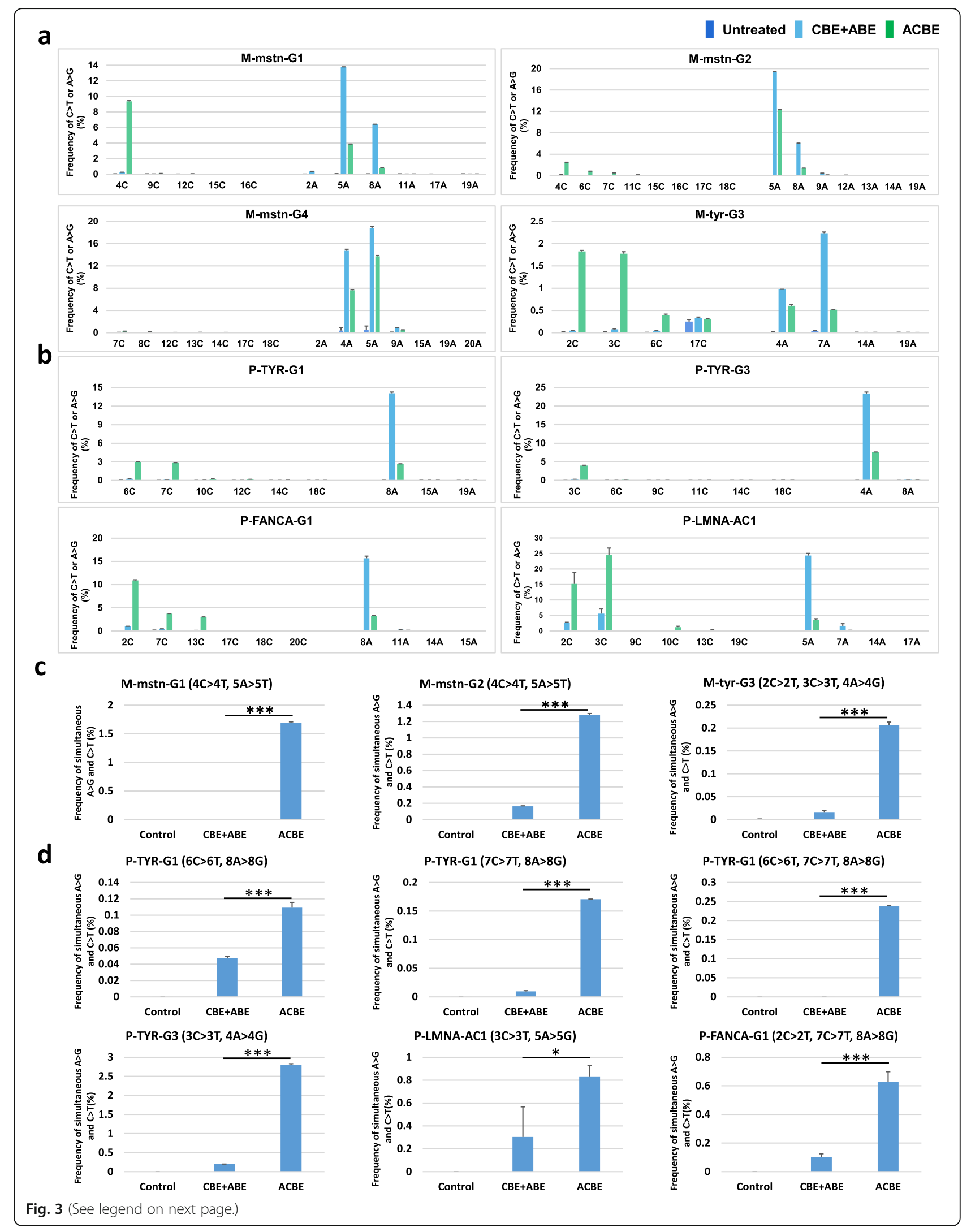


(See figure on previous page.)

Fig. 3 ACBE-mediated dual-function base editing in MEFs and PFFs. a The base editing effects of ACBE in mstn and tyr loci of MEFs; $\mathbf{b}$ The base editing effects of ACBE in TYR, FANCA, and LMNA loci of PFFs. The efficiencies of base editing $(\mathbf{a}, \mathbf{b})$ were quantified from amplicon deep sequencing. Values and error bars indicate mean \pm s.e.m. of three independent experiments. An untreated cell sample served as control. c, d The efficiencies of ACBE-mediated simultaneous C-to-T and A-to-G base substitutions in the targeting loci of MEFs (c) and PFFs (d) were calculated the reads of amplicon deep sequencing. N.S., not significant $(P>0.05) ;{ }^{*}(0.05>P \geq 0.001)$; ${ }^{* *}(0.01>P \geq 0.0001)$; ${ }^{* * *}(P<0.0001)$ (two-tailed $t$ test)

the prior C-to- $\mathrm{T}$ point mutation, whereas the $\mathrm{CBE}+$ $A B E$ system showed the prior mutation of A-to-G, which was consistent with the characteristic of base editing in HEK293 (Fig. 3a, b). The editing efficiency of Cto- $\mathrm{T}$ conversions and A-to-G conversions reached $9.35 \%$ and $13.71 \%$ for ACBE in the tested loci of MEFs, respectively (Fig. 3a), whereas $24.45 \%$ of C-to- $\mathrm{T}$ and $7.58 \%$ of A-to-G substitutions in PFFs (Fig. 3b). The editing effects of the simultaneous C-to- $\mathrm{T}$ and A-to-G point mutation at the same DNA strand were further analyzed at the M-mstn-G1, M-mstn-G2, and M-tyr-G3 targeting sites for MEFs and at the P-TYR-G1, P-TYR-G3, PFANCA-G1, and P-LMNA-AC1 targeting sites for PFFs. For these targeting loci, the ACBE system performed higher efficiency of simultaneous C-to- $\mathrm{T}$ and A-to-G point mutation at the same DNA strand than the $\mathrm{CBE}+$ $\mathrm{ABE}$ system. The efficiencies of dual-function base editing at the same DNA strand ranged from 0.21 to $1.69 \%$ for $\mathrm{ACBE}$ and 0.0015 to $0.16 \%$ for $\mathrm{CBE}+\mathrm{ABE}$ (Fig. 4c, d). These efficiencies were lower than those in HEK293 cells probably because of the low transfection frequency of primary somatic cells. Moreover, the top four POTs of M-mstn-G2, M-tyr-G3, P-TYR-G3, and P-LMNA$\mathrm{AC} 1$ were selected to verify the off-target effects of ACBE by Sanger sequencing. The Sanger sequencing results showed that no detectable off-target effects were observed in all tested POTs (Additional file 1: Fig. S8). These results indicated that the ACBE system maintained the dual function of C-to-T and A-to-G point mutations and could induce simultaneous $\mathrm{C}$-to- $\mathrm{T}$ and A-to-G substitution at the same DNA strand with high specificity in mouse and porcine primary somatic cells.

\section{Evaluation of base editing efficiency with different spacer lengths of sgRNA in the ACBE system}

As previously reported, different spacer lengths of sgRNAs influenced the base editing effects of ABE [22]. Next, the effects of different sgRNA spacer lengths on the ACBE system were evaluated. Six sgRNAs with spacer lengths of $15,18,20,24,27$, and $31 \mathrm{nt}$ (referred to as S2-15, S2-18, S2-20, S2-24, S2-27, and S2-31) targeting the exogenous EGFP were designed and analyzed in the HEK293-EGFP cell line (Fig. 4a). The ACBE-expressing vectors and EGFP-Stop2 with different spacer lengths were co-transfected into the HEK293-EGFP cells. Fiveday post transfection, the cells were collected for flow cytometry analysis. The results showed that the proportion of negative cells with S2-18 (40.27\%) was similar to S2-20 (40.50\%), whereas that of S2-15, S2-24, S2-27, and S2-31 were significantly reduced compared with S2-20 and S2-18 (Fig. 4b). Moreover, six sgRNAs with spacer lengths of 15, $18,20,24,27$, and $31 \mathrm{nt}$ targeting the endogenous P53-G8 and P53-G9 loci (referred to as P53-G8-15, 18, 20, 24, 27, and 31 and P53-G9-15, 18, 20, 24, 27, and 31) were tested in HEK293 cells (Fig. 4c, d). Consistent with the results of EGFP, the sgRNAs of the P53-G8 and P53-G9 loci with 20 nt spacer length had optimal base editing effects in the editing windows (Fig. 4e, f). The sgRNAs with $15 \mathrm{nt}$ spacer length could not support the base editing, similar to the reported effect of wild-type Cas9 [23]. The sgRNAs with longer spacer over $20 \mathrm{nt}$ reduced the base editing efficiencies of $\mathrm{C}$-to-T or A-to-G to a varying degree at the editing windows while slightly increased the base editing efficiencies at some bystander bases. In P53-G9 targeting locus, the sgRNA with $24 \mathrm{nt}$ spacer length showed the highest efficiency of C-to-T point mutation at $1 \mathrm{C}$ (Fig. $4 \mathrm{f})$. We also detected the effects of simultaneous C-to- $\mathrm{T}$ and A-to-G substitutions at the same DNA strand with different spacer lengths of sgRNAs by analyzing the corresponding read ratio of amplicon deep sequencing. The results showed that sgRNA with $20 \mathrm{nt}$ spacer length had the optimal simultaneous C-to- $\mathrm{T}$ and A-to-G substitutions at the same DNA strand $(0.006 \%$ of P53-G8-15, 0.09\% of P53-G8-18, 2.36\% of P53-G8-20, 1.58\% of P53-G8-24, 1.23\% of P53-G8-27, $1.99 \%$ of P53-G8-31, $0.017 \%$ of P53-G9-15, 0.67\% of P53G9-18, 2.41\% of P53-G9-20, 0.095\% of P53-G9-24, 0.70\% of P53-G9-27, and 1.10\% of P53-G9-31) (Fig. 4g, h). These results indicated that shortening or extending the sgRNA spacer length could significantly decrease the efficiency of simultaneous C-to-T or A-to-G point mutation at the same DNA strand.

\section{Different linker length influences the dual-function base editing effect of the ACBE system}

The linker peptide linked the components of base editors and maintained the balance of these functional domains. The length of the linker peptide might change the spatial position of ecTadA ${ }^{\mathrm{WT} / *}$, nCas9, and PmCDA1 and then affect the efficiency of dual-function base editing. Next, the base editing effects of ACBEs with different lengths of the linker peptide between ecTadA ${ }^{\mathrm{WT} /{ }^{\prime \prime}}$ and nCas9 and between nCas9 and PmCDA1 were evaluated, respectively. As shown in Fig. 5a, three ACBE variants with 0,10 , and 20 amino acid (aa) linkers between 


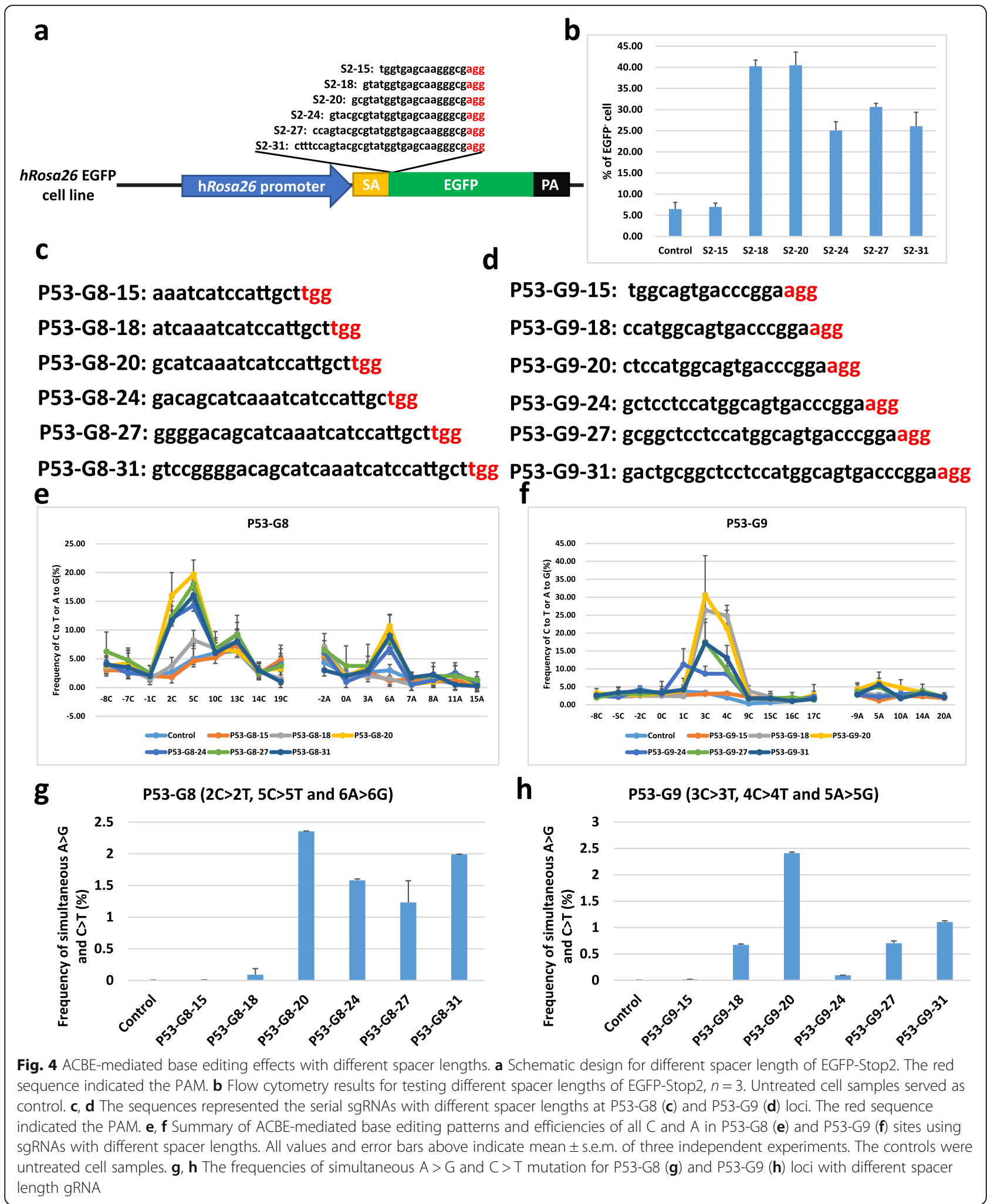

nCas9 and PmCDA1 were designed and constructed (referred to as ACBE-0C, ACBE, and ACBE-20C). The linker peptide of these $\mathrm{ACBEs}$ between ecTadA ${ }^{\mathrm{WT} / *}$ and
nCas9 was all 32 aa length. Similarly, the linker peptide between nCas9 and PmCDA1 was identical to that of 10 aa length, whereas the length between ecTadA ${ }^{\mathrm{WT} / "}$ and 


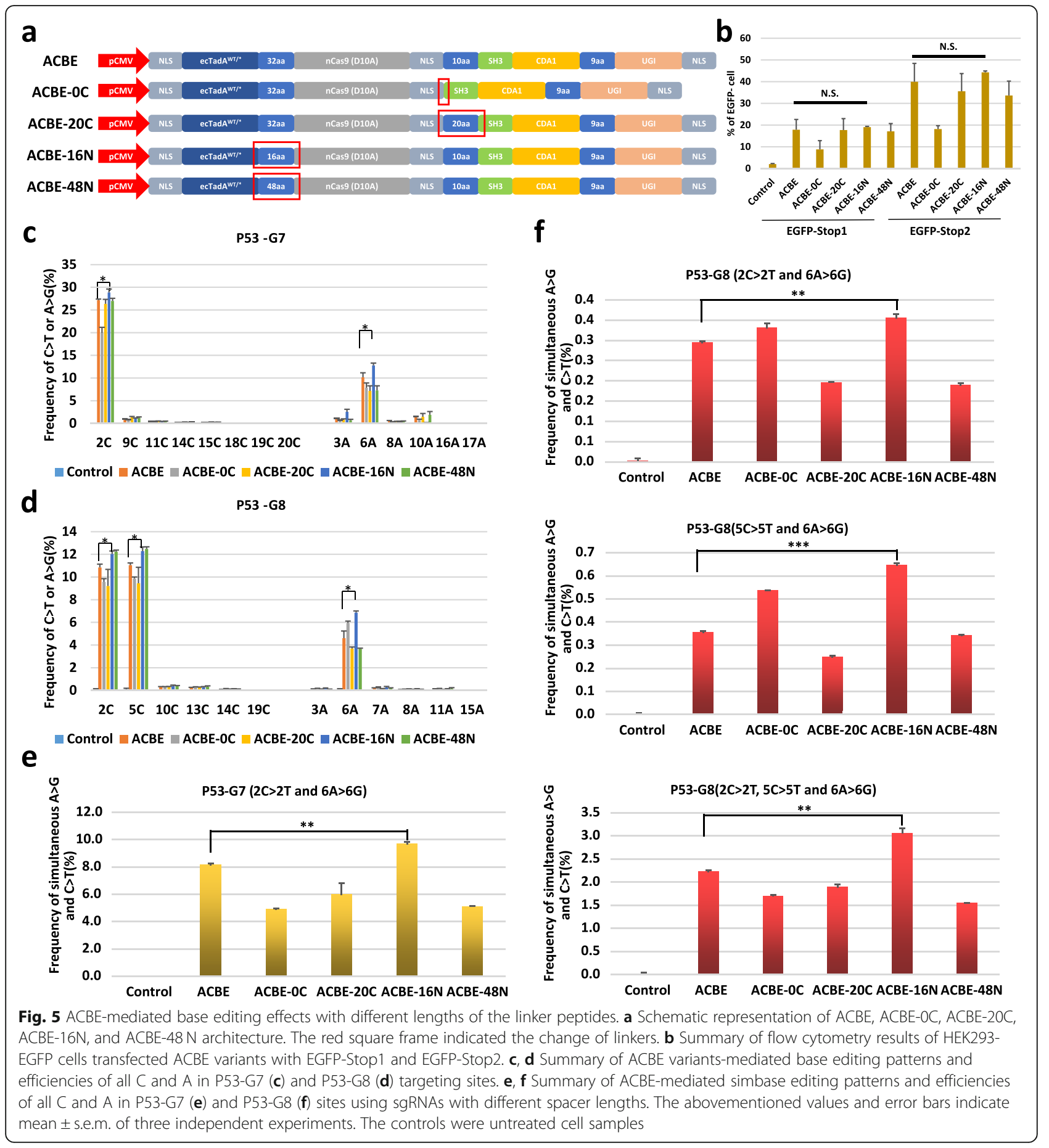

nCas9 was designed as 16,32 , and 48 aa (referred to as ACBE-16N, ACBE, and ACBE-48 N). The base editing efficiencies of these five ACBE variants were assessed at the exogenous EGFP and human endogenous TP53 genes. In the HEK293-EGFP cell line, EGFP-Stop1 for C-to-T substitution and EGFP-Stop2 for A-to-G substitution were used. Flow cytometry analysis showed that all variants maintained the base editing ability of C-to- $\mathrm{T}$ and A-to-G substitution, whereas the ACBE-10C represented a significant reduction in the efficiency of C-to- $\mathrm{T}$ and A-to-G point mutations. Moreover, the average proportion of EGFP-negative cells in the ACBE-16N group was higher than that in the ACBE group with no significant differences (Fig. 5b). For endogenous TP53 genes, P53-G7 and P53-G8 were tested. The amplicon deep sequencing results showed that different variants had 
different C-to-T and A-to-G point mutations. Notably, ACBE-16N showed more efficient base editing than ACBE in C-to-T and A-to-G substitutions in two targeting loci with a significant difference, whereas other variants showed a decreased efficiency in C-to- $\mathrm{T}$ and A-to$G$ point mutations or slightly increased efficiency in Cto-T or A-to-G mutation compared with ACBE (Fig. 5c, d). Furthermore, we evaluated whether ACBE variants could promote the simultaneous C-to- $\mathrm{T}$ and A-to-G point mutations at the same DNA strand. As shown in Fig. 5e, f, ACBE-16N could significantly increase the efficiency of heterogeneous mutation at the same DNA strand in P53-G7 $(2 \mathrm{C}>2 \mathrm{~T}$ and $6 \mathrm{~A}>6 \mathrm{G}, 8.178 \%$ of ACBE and $9.696 \%$ of ACBE-16N) and P53-G8 $(2 \mathrm{C}>2 \mathrm{~T}$ and $6 \mathrm{~A}>6 \mathrm{G}, 0.295 \%$ of $\mathrm{ACBE}$ and $0.356 \%$ of $\mathrm{ACBE}-$ $16 \mathrm{~N} ; 5 \mathrm{C}>5 \mathrm{~T}$ and $6 \mathrm{~A}>6 \mathrm{G}, 0.357 \%$ of $\mathrm{ACBE}$ and $0.646 \%$ of $\mathrm{ACBE}-16 \mathrm{~N}$; and $2 \mathrm{C}>2 \mathrm{~T}, 5 \mathrm{C}>5 \mathrm{G}$, and $6 \mathrm{~A}>6 \mathrm{G}$, $2.229 \%$ of $\mathrm{ACBE}$ and $3.061 \%$ of $\mathrm{ACBE}-16 \mathrm{~N}$ ) targeting loci. ACBE-OC could partially increase the efficiency of a simultaneous point mutation at the same DNA strand in two dual base editing patterns $(2 \mathrm{C}>2 \mathrm{~T}$ and $6 \mathrm{~A}>6 \mathrm{G}$, and $5 \mathrm{C}>5 \mathrm{~T}$ and $6 \mathrm{~A}>6 \mathrm{G}$ ) of P53-G8, whereas ACBE$20 \mathrm{C}$ and $\mathrm{ACBE}-48 \mathrm{~N}$ did not show any improvement in simultaneous C-to- $\mathrm{T}$ and A-to-G point mutations at the same DNA strand (Fig. 5e, f). These results showed that changing the length of linkers could regulate the efficiency of C-to-T and A-to-G substitutions of the ACBE system, which provides a strategy to optimize the dual function of the ACBE system.

\section{Discussion}

Previous reports showed that the dual-function base editors, STEMEs [16] and Target-ACE [17], could be used for simultaneous C-to- $\mathrm{T}$ and A-to-G base editing. In addition, during the revision process of this work, three independent studies reported that base editors fused with $\mathrm{CBE}$ and $\mathrm{ABE}$ (referred to as synchronous programmable adenine and cytosine editor (SPACE) [24], A\&C-BEmax [25], Target-ACEmax [17] (previously posted in bioRxiv)). However, the abovementioned systems have only been evaluated in either plant cells or mammalian immortalized cells and remained to be tested in mammalian primary somatic cells.

In this study, using complementary structure features in a position of deaminases and editing windows between Target-AID and ABE7.10, we fused the two base editors and generated a new base editing tool, namely, the ACBE system. ACBE had the similar nucleotide position spectrum (+ 1 to $+7 \mathrm{bp})$ compared with TargetAID [11], whereas had narrowed editing spectrum $(+4$ to + $6 \mathrm{bp}$ ) compared with ABE7.10 [12]. The effectiveness test of simultaneous C-to-T and A-to-G substitutions in seven endogenous genes with the ACBE system showed that more than half $(25 / 43)$ of sgRNAs, which covered both cytosine and adenine, could simultaneously achieve C-to- $\mathrm{T}$ and A-to-G conversions. The other 18 sgRNAs resulted in only C-to-T base editing, but no Ato-G conversion, indicating that the frequency of A-to-G conversion was lower than that of $\mathrm{C}$-to- $\mathrm{T}$ conversion in the ACBE system, which was opposite to that when $\mathrm{CBE}+\mathrm{ABE}$ was applied for base editing. PmCDA1 has a greater affinity of a single-strand DNA than TadA/ TadA* [26, 27], meaning that it also could bind to nonspecific single-strand DNA more frequently, which has been used to explain why the CBE system would lead to much higher DNA random off-target than ABE system $[28,29]$. Therefore, in $C B E+A B E$ system, the higher DNA binding ability of PmCDA1 make some of PmCDA1 bind to the nonspecific single DNA strand randomly, which would reduce the number of PmCDA1 protein molecules to bind the desired target site, thus $\mathrm{C}$ to- $\mathrm{T}$ conversion frequency is lower than that of A-to-G conversion in $\mathrm{CBE}+\mathrm{ABE}$ system. However, in the $\mathrm{ACBE}$ system, PmCDA1 and TadA/TadA* were constructed in the same vector linked with nCas9, which made them have the same chance to present at the targeting site. Due to the higher DNA binding ability, PmCDA1 is easier to occupy the single DNA strand at the R-loop created by nCas9, resulting in a prior C-to- $\mathrm{T}$ to $\mathrm{A}-$ to- $\mathrm{G}$ in ACBE-mediated base editing. The unequal ability of DNA binding between the two deaminases reduced the overall efficiency of simultaneous C-to- $\mathrm{T}$ and A-to-G substitution in the ACBE system. Thus, the modification of PmCDA1 to reduce the DNA binding ability might be a potential approach to increase the efficiency of heterologous base editing at the same locus.

The somatic cell gene editing, followed by nuclear transfer, has become a routine approach to generate genetically modified animals. Our results showed that the ACBE system was also effective in MEFs and PFFs in creating dual conversions of C-to $\mathrm{T}$ and A-to-G. The overall gene editing effect was slightly lower than that of HEK293 cells, and this finding was reasonable because primary somatic cells had a limited ability to proliferate in vitro culture and a lower capacity to accept gene transfection compared with immortalized cell lines. The efficiency of the ACBE system for the simultaneous generation of C-to-T and A-to-G mutations in somatic cells was acceptable because it could produce sufficient cells for making genetically modified animals with heterogeneous base editing through a somatic cell nuclear transfer approach [30].

ACBEs with different lengths of linkers or sgRNAs had different base editing efficiencies in the exogenous and endogenous targeting loci. ACBE with 16 aa linker between ecTadA ${ }^{\mathrm{WT} / *}$ and nCas9 had the optimal dualfunctional base editing, possibly because 16 aa linker made the deaminases closer to targeting DNA, thereby 
increasing the base editing efficiency. In addition, similar to wild-type Cas9 and single-function base editors, $20 \mathrm{nt}$ sgRNA had the highest simultaneous C-to- $\mathrm{T}$ and A-to$\mathrm{G}$ base editing. Thus optimization of linkers and sgRNAs was a potential approach for increasing the efficiency of heterologous base editing at the same locus.

In this report, the ACBE system was successfully constructed by integrating two basic editors, which require NGG PAM. Some existing strategies could be used to expand the targeting scope of ACBE because previous studies have developed many variants of CRISPR-Cas protein requiring different PAM sequences or the restricted-less PAMs [31, 32]. Recently, C-to-G base editors consisted of a Cas9 nickase, a cytidine deaminase variant, and uracil DNA N-glycosylase (eUNG), which could induce targeted C-to-G transversions with a high editing specificity in mammalian cells [33, 34]. Similarly, new dual-function base editors could be generated to simultaneously induce C-to-G and A-to-G by using the strategies established in this study.

The off-target effect of a new gene-editing tool should be considered. As the components of the ACBE system were all derived from the existing single base editing system, the ACBE system might contain the off-target characteristics of the componential base editor, including the DNA and RNA off-target editing ability. In this study, we found that the proportion of indels and the DNA potential off-target effects in the ACBE system did not increase compared with that of the single base editor. The DNA random off-target and RNA off-target effects of the ACBE system were not detected in this study, which should be tested in future research.

\section{Conclusion}

The ACBE system integrating the Target-AID and ABE7.10 into a single structure obtained the dual function of C-to-T and A-to-G substitutions and could efficiently induce heterogeneous base mutations at the same DNA strand with single sgRNA in immortalized and primary somatic cells. The application of the ACBE system would expand base editor toolkits and should promote the generation of gene editing animals and the gene therapy of genetic diseases with heterogeneous point mutations.

\section{Methods}

\section{Vector construction}

Plasmids expressing Target-AID (pcDNA3.1-TargetAID) and ABE7.10 (pcDNA3.1-ABE7.10) were obtained from our lab. The pcDNA3.1-ACBE expressing plasmid was constructed by cloning the PmCDA1 and UGI fragments amplified from pcDNA-Target-AID into the $\mathrm{C}$ terminus of plasmid pcDNA3.1-ABE7.10. The
pcDNA3.1-ACBE-0C and pcDNA3.1-ACBE-20C were constructed similarly to pCDNA3.1-ACBE but with the 0 and 20 aa linkers, respectively. pcDNA3.1-ACBE-16N and pCDNA3.1-48 $\mathrm{N}$ were constructed by fusing the heterodimer of TadA with 16 and 48 aa linkers, respectively, into the $\mathrm{N}$ terminus of pcDNA3.1-Target-AID. These vectors were generated by using a recombination kit (ClonExpress ${ }^{\circ}$ MultiS, Vazyme). All sgRNAs used in this study were designed in accordance with the G-N19NGG rule. In brief, two complementary oligonucleotides of sgRNAs were synthesized and then annealed to double-stranded DNAs. The annealed products were then cloned into the BbsI-digested U6-sgRNA cloning vector, and the sgRNA-expressing plasmids were obtained. All newly constructed plasmids were confirmed by Sanger sequencing.

\section{Cell culture and transfection}

HEK293 cells were cultured in Dulbecco's modified Eagle's medium (DMEM, HyClone) supplemented with $10 \%$ fetal bovine serum (FBS, Gibco), whereas the PFFs and MEFs were cultured in DMEM supplemented with $15 \%$ FBS, $1 \%$ nonessential amino acids (NEAA, Gibco), $2 \mathrm{mM}$ GlutaMAX (Gibco), and $1 \mathrm{mM}$ sodium pyruvate (Gibco). The culture dishes of HEK293 and MEFs were maintained at $37.5^{\circ} \mathrm{C}$ with $5 \% \mathrm{CO}_{2}$, and PFFs were maintained at $38.5^{\circ} \mathrm{C}$ with $5 \% \mathrm{CO}_{2}$.

Before electroporation, the cells were digested with $0.05 \%$ pancreatin (Gibco) and collected. Then, the collected cells were co-electroporated sgRNA-expressing vectors $(4 \mu \mathrm{g})$ with pcDNA3.1-Target-AID $(7.2 \mu \mathrm{g})$, pcDNA3.1-ABE7.10 (7.2 $\mu \mathrm{g})$, pcDNA3.1-Target-AID+ pcDNA3.1-ABE7.10 $(7.2 \mu \mathrm{g}+7.2 \mu \mathrm{g})$ or pCDNA3.1ACBE $(8 \mu \mathrm{g})$ at $1110 \mathrm{~V}, 30 \mathrm{~ms}$, and 2 pulse (HEK293) or $1350 \mathrm{~V}, 30 \mathrm{~ms}$, and 1 pulse (PFFs, MEFs) by using the Neon $^{\text {tw }}$ transfection system (Life Technology). The transfected cells were collected and sequenced 3 days later to detect the efficiency of base editing in genome. In addition, transfected HEK293-EGFP cells were detected with flow cytometry 5 days post-transfection by using BD Accuri C6.

\section{DNA extraction}

Whole DNAs were extracted from the cells by using two approaches. (1) Few cells were collected and lysed in $10 \mu \mathrm{L}$ of lysis buffer $(0.45 \% \mathrm{NP}-40$ plus $0.6 \%$ proteinase K) at $56{ }^{\circ} \mathrm{C}$ for $60 \mathrm{~min}$ and then at $95^{\circ} \mathrm{C}$ for $10 \mathrm{~min}$. (2) For a large amount of cells, genomic DNAs were extracted by using a TIANamp Genomic DNA kit (TIAN GEN) in accordance with the manufacturer's instructions. The lysate or extracted DNA was then used as a PCR template. 


\section{TA clone sequencing}

The target sequence was first generated from the genome of transfected cells by PCR with the $2 \times$ Phanta Max Master mix (Vazyme) and specific primer. Then, an adenine deoxyribonucleotide was added to the PCR products with Taq polymerase at the $72{ }^{\circ} \mathrm{C}$ for $10 \mathrm{~min}$ $(10 \mu \mathrm{L}$ reaction mix, consisting of $5 \mu \mathrm{L} 2 \times$ Rapid Taq Master Mix (Vazyme) and $5 \mu \mathrm{L}$ PCR product). The final PCR product was ligased to pMD18-T Vector (TaKaRa) with Solution I (TaKaRa) at $16^{\circ} \mathrm{C}$ for $1 \mathrm{~h}(10 \mu \mathrm{L}$ reaction mix, consisting of $4.5 \mu \mathrm{L}$ final PCR product, $0.5 \mu \mathrm{L}$ pMD18-T Vector and $5 \mu \mathrm{L}$ Solution I). The ligation product was transfected to $50 \mu \mathrm{L}$ DH $5 \alpha$ cells and cultured at $37^{\circ} \mathrm{C}$ until the proper single bacteria colony occurred. Ten single bacterial colonies were sent for vector extraction and then Sanger sequencing.

\section{Amplicon deep sequencing and data analysis}

The detecting target region was initially amplified through PCR from the extracted DNA of the transfected cells with the corresponding site-specific primers. Each PCR was performed in $20 \mu \mathrm{L}$ volume comprising $1 \mu \mathrm{L}$ of the template, $1 \mu \mathrm{L}$ of $10 \mu \mathrm{M}$ each primer, and $10 \mu \mathrm{L} 2 \times$ Phanta master mix with the following thermal cycler conditions: $95^{\circ} \mathrm{C}$ for $3 \mathrm{~min} ; 12$ cycles of $95^{\circ} \mathrm{C}$ for $10 \mathrm{~s}$, $55^{\circ} \mathrm{C}$ for $10 \mathrm{~s}$, and $72{ }^{\circ} \mathrm{C}$ for $10 \mathrm{~s}$; followed by $72{ }^{\circ} \mathrm{C}$ for 5 min as a final extension; and $12^{\circ} \mathrm{C}$ for hold. The products of the first reaction were used as the PCR template, and the second PCR amplification was performed in $40 \mu \mathrm{L}$ volume containing $2 \mu \mathrm{L}$ of the template and the custom Illumina index primers with the following thermal cycler conditions: $95^{\circ} \mathrm{C}$ for $3 \mathrm{~min} ; 30$ cycles of $95^{\circ} \mathrm{C}$ for $10 \mathrm{~s}, 55^{\circ} \mathrm{C}$ for $10 \mathrm{~s}$, and $72{ }^{\circ} \mathrm{C}$ for $10 \mathrm{~s} ; 72{ }^{\circ} \mathrm{C}$ for $5 \mathrm{~min}$ as a final extension; and $12{ }^{\circ} \mathrm{C}$ for hold. All the amplified products in different target regions were electrophoresed in $1.5 \%$ agarose gels with $1 \times \mathrm{TAE}$ buffer, and the target bands were cut for extraction by using a HiPure Gel Pure DNA Mini kit (Magen). The concentration of purified products was quantified with an Ipure Qubit dsDNA HS assay kit (IGE Biotech). Then, equivalent amounts of the purified products were mixed to produce an Illumina sequencing library. The library was sent to IGE Biotech (Guangzhou) for amplicon deep sequencing by using an Illumina HiSeq 2500 platform. The protospacer sequences in the reads were investigated to identify C-toT, A-to-G, simultaneous C-to- $\mathrm{T}$ and A-to-G point mutations, and indels. The presented ratio was calculated by comparing the individual reads to the whole reads. The amplicons were sequenced in three of the repeated assays for each target site. Amplicon reads with a quality score of $<30$ were filtered.

\section{Off-target analysis}

The POTs of each sgRNA were predicted to analyze site-specific edits by the base editing systems via an online design tool (http://www.rgenome.net/cas-offinder/) [35] allowing for un-gapped alignment with up to three mismatches in the sgRNA target sequence. All POTs were amplified via PCR and then subjected to Sanger sequencing to confirm the off-target effects.

\section{Statistical analysis}

Data were statistically analyzed by using GraphPad Prism v.7.0 and Excel. The average editing frequencies of different cytosines and adenines in the editing window were analyzed on the online tool, EditR 1.0.9 (https://moriaritylab.shinyapps.io/editr_v10/) [21]. The numerical values of target sites were presented as means \pm s.e.m. or means.

\section{Supplementary information}

Supplementary information accompanies this paper at https://doi.org/10. 1186/s12915-020-00866-5.

\begin{abstract}
Additional file 1: Figure S1-S8. Figure S1-[Detecting the expression of base editors]. Figure S2-[The ACBE system mediated heterologous Cto-T plus A-to-G substitutions in HEK293-EGFP cells]. Figure S3-[The preference analysis of ACBE]. Figure S4-[Comparation of different base editor-mediated base editing patterns and efficiencies of all $\mathrm{C}$ and $\mathrm{A}$ in P53-G7, P53-G8, LMNA-G1, LDHA-G1 and PGK1-G1 targeting sites]. Figure S5-[Summary of mutation patterns and efficiencies of cells transfected CBE or ABE with P53-G7, P53-G8, LMNA-G1, LDHA-G1 and PGK1-G4, respectively]. Figure S6-[Off-target analysis of different base editing system]. Figure S7 [Off-target analysis of ACBE system]. Figure S8-[Offtarget analysis of ACBE system in MEFs and PFFs].
\end{abstract}

Additional file 2 : Tables S1-S2. TableS1-[sgRNAs used in this study]. TableS2-[The nucleotides in positions 4-6 of 18 sgRNAs with only C-to-T conversions mediated by the ACBE system].

\section{Acknowledgements}

We thank all the members in the laboratory of Prof. Liangxue Lai.

\section{Authors' contributions}

KW and LL conceived, designed, and supervised the project. JX and XH designed and performed the experiments and analyzed data. SG analyzed the deep sequencing data. XW, YL, FC, NL, ZO, QZ, WG, QJ, HS, ZZ, XZ, ML, $J W, Y Y, L Q$, and HW participated in plasmids construction, cell culture, and discussion. KW, LL, and JX wrote the paper. All authors have read and approved the final manuscript.

\section{Funding}

This work was financially supported by National Key Research and Development Program of China (2017YFA0105103), National Natural Science Foundation of China (81941004, 81702115, 81672317), Key Research \&

Development Program of Guangzhou Regenerative Medicine and Health Guangdong Laboratory (2018GZR110104004), the Youth Innovation Promotion Association of the Chinese Academy of Sciences (2019347), Science and Technology Planning Project of Guangdong Province, China (2017B020231001, 2017B030314056), Science and Technology Program of Guangzhou, China (201704030034, 201907010039, 202007030003, 202002030382), and Research Unit of Generation of Large Animal Disease Models, Chinese Academy of Medical Sciences (2019-12M-5-025).

\section{Availability of data and materials}

The high-throughput sequencing data are available at the Sequence Read Archive (PRJNA601637) of the NCBI [36]. The authors declare that all data 
supporting the findings of this study are available in the article and its supplementary figures and tables. Such data are also available from the corresponding author upon request.

\section{Ethics approval and consent to participate} Not applicable.

\section{Competing interests}

The authors declare that they have no competing interests.

\section{Author details}

${ }^{1}$ CAS Key Laboratory of Regenerative Biology, Guangdong Provincial Key Laboratory of Stem Cell and Regenerative Medicine, Guangzhou Institutes of Biomedicine and Health, Chinese Academy of Sciences, Guangzhou 510530, China. ${ }^{2}$ Research Unit of Generation of Large Animal Disease Models, Chinese Academy of Medical Sciences (2019RU015), Guangzhou 510530, China. ${ }^{3}$ University of Chinese Academy of Sciences, Beijing 100049, China. ${ }^{4}$ Bioland Laboratory (Guangzhou Regenerative Medicine and Health Guangdong Laboratory, GRMH-GDL), Guangzhou 510005, China. ${ }^{5}$ Institute of Physical Science and Information Technology, Anhui University, Hefei 230601, China.

Received: 1 April 2020 Accepted: 9 September 2020

Published online: 23 September 2020

\section{References}

1. Gaj T, Gersbach CA, Barbas CF. ZFN, TALEN, and CRISPR/Cas-based methods for genome engineering. Trends Biotechnol. 2013;31(7):397-405.

2. Chaudhary R, Singh B, Kumar M, Gakhar SK, Saini AK, Parmar VS, Chhillar AK. Role of single nucleotide polymorphisms in pharmacogenomics and their association with human diseases. Drug Metab Rev. 2015;47(3):281-90.

3. Landrum MJ, Lee JM, Benson M, Brown G, Chao C, Chitipiralla S, Gu B, Hart J, Hoffman D, Hoover J, et al. ClinVar: public archive of interpretations of clinically relevant variants. Nucleic Acids Res. 2016;44(D1):D862-868.

4. Sun YV, Hu YJ: Integrative analysis of multi-omics data for discovery and functional studies of complex human diseases. Adv Genet. 2016;93:147-90.

5. Rothschild MF, Hu ZL, Jiang ZH. Advances in QTL mapping in pigs. Int J Biol Sci. 2007;3(3):192-97.

6. Shimatani Z, Kashojiya S, Takayama M, Terada R, Arazoe T, Ishii H, Teramura $\mathrm{H}$, Yamamoto T, Komatsu H, Miura K, et al. Targeted base editing in rice and tomato using a CRISPR-Cas9 cytidine deaminase fusion. Nat Biotechnol. 2017:35(5):441-3.

7. Molla KA, Shih J, Yang Y. Single-nucleotide editing for zebra3 and wsl5 phenotypes in rice using CRISPR/Cas9-mediated adenine base editors. aBIOTECH. 2020;1(2):106-18.

8. Molla KA, Yang YN. CRISPR/Cas-Mediated Base editing: technical considerations and practical applications. Trends Biotechnol. 2019;37(10): 1121-42.

9. Rees HA, Liu DR. Base editing: precision chemistry on the genome and transcriptome of living cells. Nat Rev Genet. 2018;19(12):770-88.

10. Komor AC, Kim YB, Packer MS, Zuris JA, Liu DR. Programmable editing of a target base in genomic DNA without double-stranded DNA cleavage. Nature. 2016;533(7603):420-4.

11. Nishida K, Arazoe T, Yachie N, Banno S, Kakimoto M, Tabata M, Mochizuki M, Miyabe A, Araki M, Hara KY, et al. Targeted nucleotide editing using hybrid prokaryotic and vertebrate adaptive immune systems. Science. 2016; 353(6305):aaf8729.

12. Gaudelli NM, Komor AC, Rees HA, Packer MS, Badran AH, Bryson DI, Liu DR. Programmable base editing of A. T to G. C in genomic DNA without DNA cleavage. Nature. 2017;551(7681):464-71.

13. Anzalone AV, Randolph PB, Davis JR, Sousa AA, Koblan LW, Levy JM, Chen PJ, Wilson C, Newby GA, Raguram A, Liu DR. Search-and-replace genome editing without double-strand breaks or donor DNA. Nature. 2019;576(7785): 149-57.

14. Lin Q, Zong Y, Xue C, Wang S, Jin S, Zhu Z, Wang Y, Anzalone AV, Raguram $A$, Doman $J$, et al. Prime genome editing in rice and wheat. Nat Biotechnol. 2020:38(5):582-5.

15. Liu Y, Li XY, He ST, Huang SH, Li C, Chen YL, Liu Z, Huang XX, Wang XL. Efficient generation of mouse models with the prime editing system. Cell Discov. 2020;6:27.
16. Li C, Zhang R, Meng XB, Chen S, Zong Y, Lu CJ, Qiu JL, Chen YH, Li JY, Gao CX. Targeted, random mutagenesis of plant genes with dual cytosine and adenine base editors. Nat Biotechnol. 2020;38(7):875-82.

17. Sakata RC, Ishiguro S, Mori H, Tanaka M, Tatsuno K, Ueda H, Yamamoto S, Seki M, Masuyama N, Nishida K, et al. Base editors for simultaneous introduction of C-to-T and A-to-G mutations. Nat Biotechnol. 2020;38(7): 865-9.

18. Wu H, Liu QS, Shi H, Xie JK, Zhang QJ, Ouyang Z, Li N, Yang Y, Liu ZM, Zhao $Y$, et al. Engineering CRISPR/Cpf1 with tRNA promotes genome editing capability in mammalian systems. Cell Mol Life Sci. 2018;75(19):3593-607.

19. Liu Z, Lu ZY, Yang G, Huang SS, Li GL, Feng SJ, Liu YJ, Li JN, Yu WX, Zhang $Y$, et al. Efficient generation of mouse models of human diseases via ABEand BE-mediated base editing. Nat Commun. 2018;9(1):2338.

20. Stoltzfus CR, Barnett LM, Drobizhev M, Wicks G, Mikhaylov A, Hughes TE, Rebane A. Two-photon directed evolution of green fluorescent proteins. Sci Rep. 2015;5:11968.

21. Kluesner MG, Nedveck DA, Lahr WS, Garbe JR, Abrahante JE, Webber BR, Moriarity BS. EditR: a method to quantify base editing from Sanger sequencing. Crispr j. 2018;1(3):239-50.

22. Ryu SM, Koo T, Kim K, Lim K, Baek G, Kim ST, Kim HS, Kim DE, Lee H, Chung E, Kim JS. Adenine base editing in mouse embryos and an adult mouse model of Duchenne muscular dystrophy. Nat Biotechnol. 2018;36(6):536-39.

23. Dahlman JE, Abudayyeh OO, Joung J, Gootenberg JS, Zhang F, Konermann S. Orthogonal gene knockout and activation with a catalytically active Cas 9 nuclease. Nat Biotechnol. 2015;33(11):1159-61.

24. Grunewald J, Zhou RH, Lareau CA, Garcia SP, lyer S, Miller BR, Langner LM, Hsu JY, Aryee MJ, Joung JK. A dual-deaminase CRISPR base editor enables concurrent adenine and cytosine editing. Nat Biotechnol. 2020;38(7):861-64

25. Zhang XH, Zhu BY, Chen L, Xie L, Yu WS, Wang Y, Li LX, Yin SM, Yang L, Hu $H D$, et al. Dual base editor catalyzes both cytosine and adenine base conversions in human cells. Nat Biotechnol. 2020;38(7):856-60.

26. Chaudhuri J, Tian M, Khuong C, Chua K, Pinaud E, Alt FW. Transcriptiontargeted DNA deamination by the AID antibody diversification enzyme. Nature. 2003;422(6933):726-30.

27. Bransteitter R, Pham P, Scharff MD, Goodman MF. Activation-induced cytidine deaminase deaminates deoxycytidine on single-stranded DNA but requires the action of RNase. Proc Natl Acad Sci U S A. 2003;100(7):4102-7.

28. Jin S, Zong Y, Gao Q, Zhu ZX, Wang YP, Qin P, Liang CZ, Wang DW, Qiu J, Zhang F, Gao CX. Cytosine, but not adenine, base editors induce genomewide off-target mutations in rice. Science. 2019;364(6437):292-5.

29. Zuo EW, Sun YD, Wei W, Yuan TL, Ying WQ, Sun H, Yuan LY, Steinmetz LM, Li YX, Yang H. Cytosine base editor generates substantial off-target singlenucleotide variants in mouse embryos. Science. 2019;364(6437):289-92.

30. Xie JK, Ge WK, Li N, Liu QS, Chen FB, Yang XY, Huang XY, Ouyang Z, Zhang QJ, Zhao $Y$, et al. Efficient base editing for multiple genes and loci in pigs using base editors. Nat Commun. 2019;10(1):2852.

31. Hu JH, Miller SM, Geurts MH, Tang WX, Chen LW, Sun N, Zeina CM, Gao X, Rees HA, Lin Z, Liu DR. Evolved Cas9 variants with broad PAM compatibility and high DNA specificity. Nature. 2018;556(7699):57-63.

32. Nishimasu H, Shi X, Ishiguro S, Gao LY, Hirano S, Okazaki S, Noda T, Abudayyeh OO, Gootenberg JS, Mori H, et al. Engineered CRISPR-Cas9 nuclease with expanded targeting space. Science. 2018;361(6408):1259-62.

33. Kurt IC, Zhou RH, lyer S, Garcia SP, Miller BR, Langner LM, Grünewald J, Joung JK. CRISPR C-to-G base editors for inducing targeted DNA transversions in human cells. Nat Biotechnol. 2020. https://doi.org/10.1038/ s41587-020-0609-x. Online ahead of print.

34. Zhao DD, Li J, Li SW, Xin XQ, Hu MZ, Price MA, Rosser SJ, Bi CH, Zhang XL. Glycosylase base editors enable C-to-A and C-to-G base changes. Nat Biotechnol. 2020. https://doi.org/10.1038/s41587-020-0592-2. Online ahead of print.

35. Bae S, Park J, Kim JS. Cas-OFFinder: a fast and versatile algorithm that searches for potential off-target sites of Cas9 RNA-guided endonucleases. Bioinformatics. 2014;30(10):1473-5.

36. Xie JK, Huang XY, Wang X, Gou SX, Liang YH, Chen FB, Li N, Ouyang Z, Zhang QJ, Ge WK, et al. ACBE, a new base editor for simultaneous C-to-T and A-to-G substitutions in mammalian systems. NCBI accession PRJNA601637, https:// www.ncbi.nlm.nih.gov/bioproject/PRJNA601637. Accessed 9 Sept 2020.

\section{Publisher's Note}

Springer Nature remains neutral with regard to jurisdictional claims in published maps and institutional affiliations. 